\title{
Detection and quantification of bacterial biofilms combining high-frequency acoustic microscopy and targeted lipid microparticles
}

\author{
Pavlos Anastasiadis ${ }^{1,2,3}$, Kristina D A Mojica $^{4,5}$, John S Allen ${ }^{3^{*}}$ and Michelle L Matter ${ }^{1 *}$
}

\begin{abstract}
Background: Immuno-compromised patients such as those undergoing cancer chemotherapy are susceptible to bacterial infections leading to biofilm matrix formation. This surrounding biofilm matrix acts as a diffusion barrier that binds up antibiotics and antibodies, promoting resistance to treatment. Developing non-invasive imaging methods that detect biofilm matrix in the clinic are needed. The use of ultrasound in conjunction with targeted ultrasound contrast agents (UCAs) may provide detection of early stage biofilm matrix formation and facilitate optimal treatment.
\end{abstract}

Results: Ligand-targeted UCAs were investigated as a novel method for pre-clinical non-invasive molecular imaging of early and late stage biofilms. These agents were used to target, image and detect Staphylococcus aureus biofilm matrix in vitro. Binding efficacy was assessed on biofilm matrices with respect to their increasing biomass ranging from $3.126 \times$ $10^{3} \pm 427$ UCAs per $\mathrm{mm}^{2}$ of biofilm surface area within $12 \mathrm{~h}$ to $21.985 \times 10^{3} \pm 855$ per $\mathrm{mm}^{2}$ of biofilm matrix surface area at $96 \mathrm{~h}$. High-frequency acoustic microscopy was used to ultrasonically detect targeted UCAs bound to a biofilm matrix and to assess biofilm matrix mechanoelastic physical properties. Acoustic impedance data demonstrated that biofilm matrices exhibit impedance values (1.9 MRayl) close to human tissue (1.35 - 1.85 MRayl for soft tissues). Moreover, the acoustic signature of mature biofilm matrices were evaluated in terms of integrated backscatter (0.0278- $\left.0.0848 \mathrm{~mm}^{-1} \times \mathrm{sr}^{-1}\right)$ and acoustic attenuation (3.9 Np/mm for bound UCAs; $6.58 \mathrm{~Np} / \mathrm{mm}$ for biofilm alone).

Conclusions: Early diagnosis of biofilm matrix formation is a challenge in treating cancer patients with infection-associated biofilms. We report for the first time a combined optical and acoustic evaluation of infectious biofilm matrices. We demonstrate that acoustic impedance of biofilms is similar to the impedance of human tissues, making in vivo imaging and detection of biofilm matrices difficult. The combination of ultrasound and targeted UCAs can be used to enhance biofilm imaging and early detection. Our findings suggest that the combination of targeted UCAs and ultrasound is a novel molecular imaging technique for the detection of biofilms. We show that high-frequency acoustic microscopy provides sufficient spatial resolution for quantification of biofilm mechanoelastic properties.

Keywords: Targeted therapy, Lipid particles, Biofilm matrix, Targeted ultrasound contrast agents, Cancer, Acoustic microscopy, Molecular imaging, Microbubbles

\footnotetext{
* Correspondence: alleniii@hawaii.edu; matter@hawaii.edu

${ }^{3}$ Mechanical Engineering, University of Hawaii at Manoa, Honolulu, HI 96822,

USA

'University of Hawaii Cancer Center, Honolulu, HI 96813, USA

Full list of author information is available at the end of the article
} 


\section{Background}

Bacterial biofilms are three-dimensional extracellular matrices composed of carbohydrates, proteins and exopolysaccharides [1-6] that develop on solid-liquid or solid-air interfaces in the body [3,7]. Biofilms consist of bacterial cells and matrix proteins. The majority of biofilms contain $10 \%$ or less of bacterial cells and over $90 \%$ matrix [8]. Biofilm matrices are highly conserved dynamic structures. Initiation of a biofilm matrix occurs by a transient interaction of bacteria with a surface followed by an adhesive stage that allows for microcolony formation and a subsequent growth and maturation stage. The complexity of biofilms allows bacteria cells to survive a multitude of environments and promotes cell dispersion to colonize new areas. These matrices may form on medical devices or fragments of dead tissue [3,9-12]. Clinically, biofilms may occur during chemotherapy and infectious diseases such as endocarditis [6,13-16].

Biofilm associated infections are resistant to treatment and recur even after repeated antibiotic therapy. One primary issue is that established biofilm matrices act as diffusion barriers and actively bind up antibiotics and antibodies thereby providing increased resistance. Overall killing bacteria that are surrounded by a microbial biofilm require up to 1000 times higher concentrations of antibiotics than those without a surrounding biofilm [17-20]. Thus, detecting, treating and inhibiting biofilm formation inside the body is a key medical challenge.

Moreover, in the clinical setting antibiotic therapy efficacy is decreased in the presence of an established biofilm making early detection critical. For example, infective endocarditis may occur due to chronic infection, has a poor prognosis and is associated with high mortality rates [14-16,21]. Indeed, there are significant diagnostic challenges for endocarditis that are attributed to the inaccessibility of intra-cardiac biofilms and the non-specific nature of the clinical symptoms [22]. Although echocardiography permits non-invasive detection of biofilms [23] it has significant limitations in the detection of early biofilm matrix formation. In addition, clinical diagnosis primarily occurs after biofilm matrices are fully established, thereby significantly decreasing available treatment options. Therefore, early detection is a crucial component of diagnosis; however no current diagnostic methodology is available that clearly delineates early and late stage matrices.

Ultrasound is an effective method for imaging biofilms in vitro [24-28]. One method used to enhance biofilm detection is the addition of UCAs (encapsulated gas bubbles), which provide a unique acoustic scattering signature thereby significantly enhancing imaging capabilities [29]. Furthermore, linking a ligand to a contrast agent's outer membrane aids in UCAs binding to tissue and is crucial in delineating disease specific regions from surrounding healthy tissue [30-35].
In this study, ligand-targeted UCAs were used as a novel method for pre-clinical non-invasive molecular imaging of early and late stage biofilms. These agents were used to target and detect Staphylococcus aureus (S. aureus) biofilm formation. Binding efficacy was assessed on established biofilms as a function of surface area. A combination of acoustic and optical microscopy was used to quantify the mechanical and structural properties of a three dimensional biofilm matrix. We show that high-frequency scanning acoustic microscopy (SAM) provides sufficient high spatial resolution for imaging and quantification of biofilm thickness and mechanoelastic properties.

\section{Results}

Biofilm formation occurs when bacterial cells enter the body and attach to the underlying endothelium or tissues. Over time, biofilms form a protective three dimensional matrix that results in lower antibody efficacy in vivo (Figure 1). Biofilm surface areas were assessed by epifluorescence microscopy images of stained $S$. aureus biofilms at various time points (Figure 2A). Biofilm matrix surface area doubled during the first 12 hours after inoculation (growing from $26.85 \mathrm{~mm}^{2} \pm 6.72 \mathrm{~mm}^{2}$ to $51.7 \mathrm{~mm}^{2} \pm$ $2.12 \mathrm{~mm}^{2}$ at $12 \mathrm{~h}$ and $24 \mathrm{~h}$ respectively; $\left.\mathrm{p}<0.05\right)$. Similar growth patterns were observed through 96 hours (68.95 mm $\mathrm{mm}^{2} \pm 4.6 \mathrm{~mm}^{2}, 122.2 \mathrm{~mm}^{2} \pm 8.56 \mathrm{~mm}^{2}$ and $179.2 \mathrm{~mm}^{2} \pm 2.97 \mathrm{~mm}^{2}$ for $48 \mathrm{~h}, 72 \mathrm{~h}$ and $96 \mathrm{~h}$ respectively; $\mathrm{p}<0.05)$. These data suggest that biofilm matrices are produced over time in our in vitro culture system.

To determine whether targeted UCAs bind to a biofilm matrix in vitro, we next examined whether targeted ultrasound contrast agents (UCAs) bound to the biofilm matrix over time. We observed an increase in the binding rate of targeted UCAs to the biofilm matrix (Figure 2B). We tested whether labeled targeted UCAs were detectable upon a labeled biofilm matrix. Tetramethylrhodamine isothiocyanate (TRITC)-streptavidin conjugated UCAs (red staining) were detectable from fluorescein isothiocyanate (FITC) anti-WGA labeled matrix (green staining). At the $12 \mathrm{~h}$ time point $1.109 \times 10^{3} \pm 142$ UCAs were bound to the biofilm. The number of bound bubbles significantly increased to $3.126 \times 10^{3} \pm 427$ over the following $12 \mathrm{~h}$. Between $24 \mathrm{~h}$ and $72 \mathrm{~h}$ labeled UCAs binding increased $\left(5.042 \times 10^{3} \pm 285\right.$ UCAs at $48 \mathrm{~h}, 7.563 \times 10^{3} \pm 142$ at $72 \mathrm{~h}$; $\mathrm{p}<0.05)$. Between $72 \mathrm{~h}$ and $96 \mathrm{~h}$ a significant increase in targeted UCAs was observed $\left(7.563 \times 10^{3} \pm 142\right.$ to $21.985 \pm$ 855 at 96 h; p < 0.05) suggesting that binding increases in correlation with biofilm matrix surface area. Fluorescence images stained for $S$. aureus biofilm matrix at various time points (Figure $2 \mathrm{C}$ ) confirms that targeted UCAs bound more as the biofilm matrix increased over 96 hours.

Developing a non-invasive diagnostic method to detect biofilm matrices early (or at initial stages) would be a valuable clinical tool if the targeted agents could be detected 


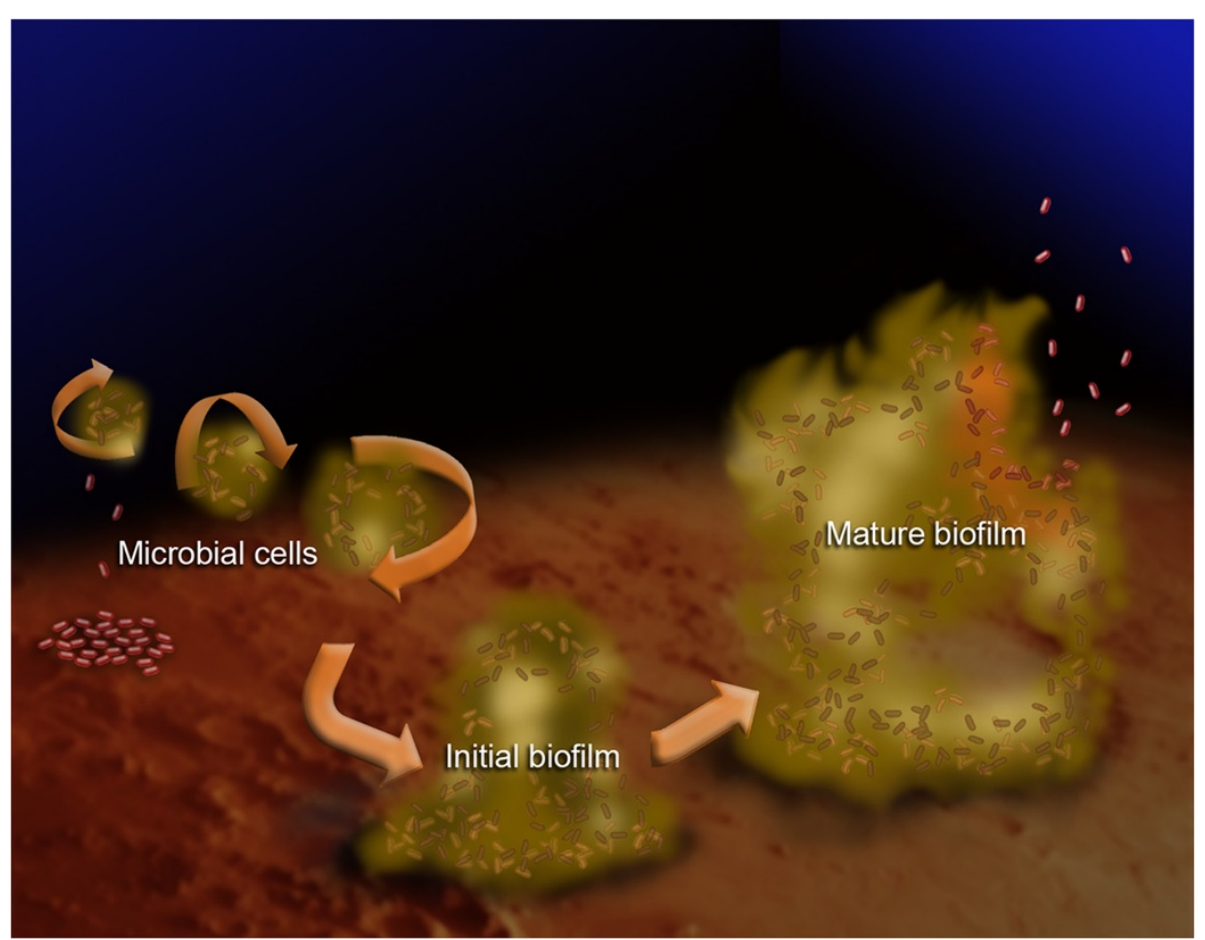

Figure 1 Biofilm matrix formation. Individual bacterial cells gain entrance into the bloodstream and attach at favorable sites. As they continue growing, they form a protective biofilm matrix against hostile agents, the immune system or fluid turbulences caused by hemodynamic forces. As the biofilm matrix matures, individual cells are dispersed into the bloodstream where they travel to distant sites in the body forming colonies. Figure adapted from [6].

acoustically. Because we determined that targeted UCAs bound proportionately to biofilm matrix mass we next assessed whether ultrasound could be used to detect targeted UCAs in vitro. The center frequency for the ultrasonic evaluation was $100 \mathrm{MHz}$ [26] allowing for a rigorous quantification of biofilm matrix mechanoelastic properties in our in vitro biofilm culture system (Table 1).

For the physical evaluation of biofilm matrix properties (density, acoustic attenuation, ultrasound velocity, acoustic impedance and bulk modulus) a time-resolved high-frequency scanning acoustic microscope was used (Fraunhofer IBMT, St. Ingbert, Germany; Table 2). For imaging, an acoustic lens is triggered by a piezoelectric transducer that emits and receives highly focused sound waves and resolves them along the time axis (Figure 3A). The echoes reflected off the sample surface, the substrate and the interface between the sample and the substrate were taken into consideration for mechanoelastic quantification. The acoustic lens is mounted on top of the stage of a Zeiss Axiovert M200, inverted light microscope (Figure 3B). This custom arrangement [36,37], where the optical microscope objective and the acoustic lens are confocally aligned allows for corresponding optical (or fluorescence) imaging and therefore facilitating novel simultaneous acoustical and optical evaluation of specimen.
S. aureus mature biofilms at day five were ultrasonically and fluorescently evaluated (Figure 4). UCAs were conjugated with TRITC-labeled streptavidin that allowed for the detection of the corresponding fluorescent signal. Because the acoustic lens is confocally aligned we were able to overlay the corresponding acoustic and fluorescent signals. In each case three or more different regions were scanned covering a total surface of $1 \mathrm{~mm}^{2}$ for each independent acquisition. For the same time point, fluorescence and optical images were acquired from the identical regions (Figure 4). The grey colored area corresponds to the acquired acoustic dataset of the biofilm matrix while the inset depicts a fluorescent image of a partial area within that ultrasonically acquired region. The epifluorescent images were comparable in terms of specimen location and provided different complementary information on the biofilm structure and mechanical properties (Table 1). Microbubbles (Targeson, San Diego, CA, USA) were 2$3 \mu \mathrm{m}$ in diameter and were detected based on fluorescence and acoustic signals (Figure 4).

We next examined whether regions of biofilm matrices can be delineated based on targeted and bound or nontargeted, non-bound UCAs. When no biofilm mass was present the targeted UCAs remain unbound as there is no ligand for them to bind to (Figure 5A). As biofilm matrix formation progresses, targeted UCAs bind to the ligand 


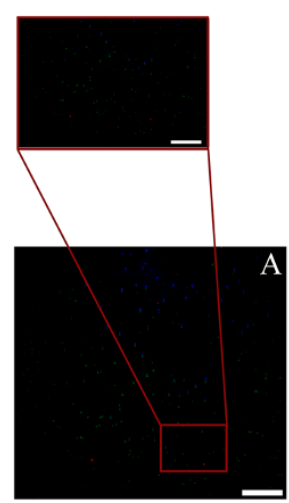

24 hours

$\mathrm{E}$
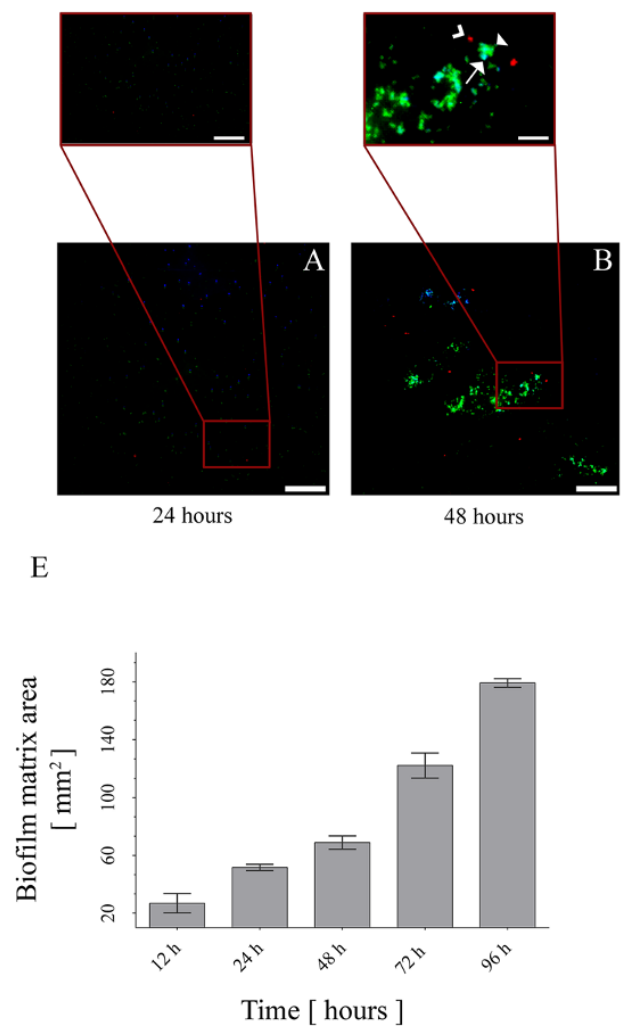

48 hours

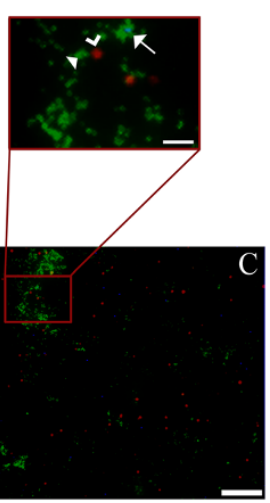

72 hours

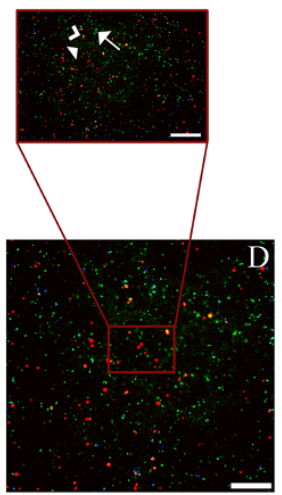

96 hours

$\mathrm{F}$

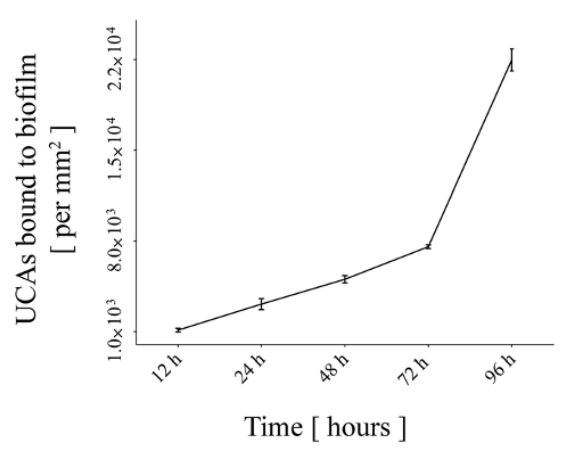

Figure 2 Targeted ultrasound contrast agents bind to biofilm matrix in a time-dependent fashion. Targeted UCAs bind to the biofilm mass. As the biofilm matrix grows, an increased surface area is accompanied by an increase in the number of bound UCAs. (A-D) Epifluorescence microscopy imaging of the biofilm matrix for 24 h, 48 h, 72 h and 96 respectively (scale bar $=50 \mu m$; scale bar of insets $=15 \mu \mathrm{m}$ ). Bacterial cells are stained with DAPI (blue; arrows), targeted UCAs are microbubbles conjugated with streptavidin (red; open arrowheads) and biofilm matrix is detected by staining for FITC-conjugated lectins (green; filled arrowheads). (E) Biofilm mass surface area over time (24 h, 48 h, 72 h and 96 h). (F) Number of targeted UCAs bound to the biofilm matrix over the same time course (24 h, 48 h, 72 h and 96 h).

present in the biofilm matrix (Figure 5B). Targeted UCAs bound to the biofilm matrix scattered sound and produced a detectable acoustic signature [38,39], which correlates with a biofilm matrix. The images shown in Figure 4 depict both the corresponding optical and acoustic images of the UCAs. The acoustic image in Figure 6A depicts UCAs reflectivity in backscatter intensity and their spatial location is depicted as red signals in the fluorescent image.

Table 1 Mechanical and elastic parameters of an S. aureus biofilm at 96 hours as determined by time-resolved high-frequency scanning acoustic microscopy

\begin{tabular}{ll}
\hline Structural and physical properties & Biofilm matrix \\
\hline Thickness $[\mu \mathrm{m}]$ & $127.23 \pm 2.87$ \\
Ultrasound velocity $[\mathrm{m} / \mathrm{s}]$ & $1523.14 \pm 12.01$ \\
Attenuation $[\mathrm{Np} / \mathrm{mm}]$ & $4.2 \pm 0.18$ \\
Acoustic impedance [MRayl] & $1.9 \pm 0.01$ \\
Density $\left[\mathrm{kg} / \mathrm{m}^{3}\right]$ & $1246.58 \pm 11.48$ \\
Bulk modulus $[\mathrm{GPa}]$ & $2.8 \pm 2.9 \times 10^{-5}$ \\
\hline
\end{tabular}

Furthermore, Figure 6A demonstrates that bound UCAs provide a stronger backscatter intensity as compared to the regions of biofilm matrix alone.

Based on linear acoustics [40] the integrated backscatter coefficient and acoustic attenuation were calculated for regions of bound and unbound UCAs. The mean integrated backscatter coefficient (IBSC; Figure 6A) was determined for frequencies ranging from $97 \mathrm{MHz}$ to $104 \mathrm{MHz}$ from

Table 2 Physical properties of the high-frequency acoustic lens used in this study

\begin{tabular}{lc}
\hline Acoustic lens properties & \\
\hline Excitation center frequency $[\mathrm{MHz}]$ & 100 \\
Max PRF [kHz] & 100 \\
Gain $[\mathrm{dB}]$ & 40 \\
Sampling rate $[\mathrm{MSamples} / \mathrm{s}]$ & 400 \\
Focal resolution $[\mu \mathrm{m}]$ & 10 \\
Aperture $[\mu \mathrm{m}]$ & 950 \\
Aperture angle $\left[^{\circ}\right]$ & 55 \\
Working distance $[\mu \mathrm{m}]$ & 900 \\
\hline
\end{tabular}



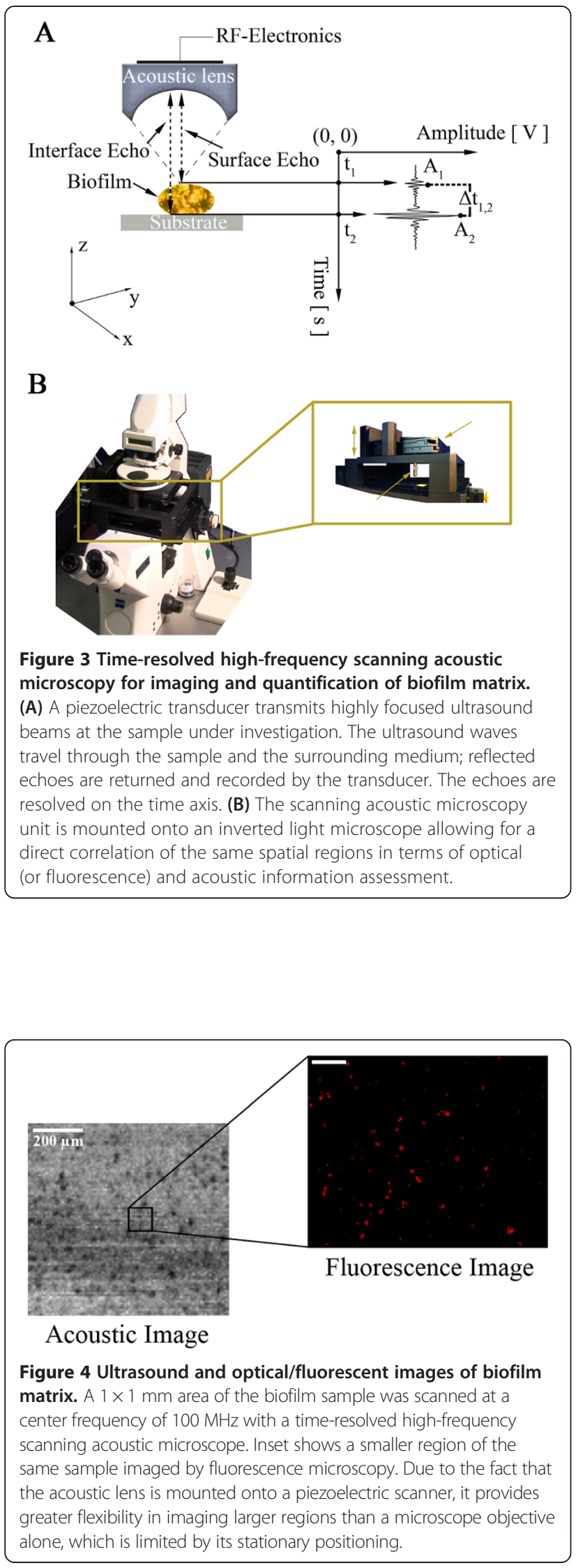

the measured biofilm regions in which targeted UCAs were bound versus biofilm matrix alone. This frequency range corresponded to 36 points at a sampling frequency of $400 \mathrm{MHz}$ while the scanning of the region of interests (ROIs) was performed with step sizes in the order of $10 \mu \mathrm{m}$ in the $\mathrm{x}$ - and $\mathrm{y}$-direction respectively. The acquired mean values of the IBSCs for the ROIs that remained bound to targeted UCAs range from $0.0278 \mathrm{~mm}^{-1} \times \mathrm{sr}^{-1}$ to $0.0848 \mathrm{~mm}^{-1} \times \mathrm{sr}^{-1}$ while the values for the standard deviation (StDev) vary from $0.0016 \mathrm{~mm}^{-1} \times \mathrm{sr}^{-1}$ to $0.0043 \mathrm{~mm}^{-1} \times \mathrm{sr}^{-1}$. The evaluation of the ROIs corresponding to the matrix without UCAs yielded for the IBSCs mean values in the range from $0.0167 \mathrm{~mm}^{-1} \times \mathrm{sr}^{-1}$ to $0.0694 \mathrm{~mm}^{-1} \times \mathrm{sr}^{-1}$ with StDev values ranging from $0.0012 \mathrm{~mm}^{-1} \times \mathrm{sr}^{-1}$ to $0.0024 \mathrm{~mm}^{-1} \times \mathrm{sr}^{-1}$ respectively.

The same ROIs used for the quantification of the IBSC were further evaluated with regard to sound attenuation (Figure 6B). ROIs were analyzed in which UCAs were either bound to the matrix or not bound. Each of the fifteen ROIs per condition consisted of nine pixels corresponding to 135 raw radio-frequency (RF) time-signals for the UCA ROIs and similarly fifteen ROIs for the matrix ROIs corresponding to another 135 raw RF time-signals. The frequency-dependent attenuation was calculated over the frequency range from $97 \mathrm{MHz}$ to $104 \mathrm{MHz}$. Equivalent to our IBSC findings this frequency range consisted of 36 points at a sampling frequency of $400 \mathrm{MHz}$. The attenuation graphs for the ROIs with bound targeted UCAs and the ROIs with plain matrix over the selected frequency range are shown in Figure 6B. Taken together, detection of targeted bound UCAs is significant compared with unbound UCAs. Our data highlight the potential of targeted UCAs as a means of molecular imaging to detect the early stages of biofilm matrix formation.

\section{Conclusions}

In this study, we report for the first time a combined optical and acoustic imaging method of infectious biofilm matrices. Ligand-targeted UCAs were used as a novel method for pre-clinical non-invasive molecular imaging of early to late stage biofilms. These agents were used to target $S$. aureus biofilm formation and assess the binding efficacy on early to late stage biofilm matrices with respect to their surface area. A combination of acoustic and optical microscopy was used to quantify $S$. aureus biofilm mechanoelastic properties. We show that time-resolved high-frequency SAM is a viable method for ultrasonic imaging in addition to quantifying mechanical and elastic properties in soft materials (eg. tissues, cells, biofilm matrices) in a non-invasive setting. Moreover, the use of targeted UCAs with highfrequency SAM allow for UCAs detection at higher frequencies other than their resonance frequency. The mechanoelastic properties of the $S$. aureus biofilm matrix are summarized in Table 1. 

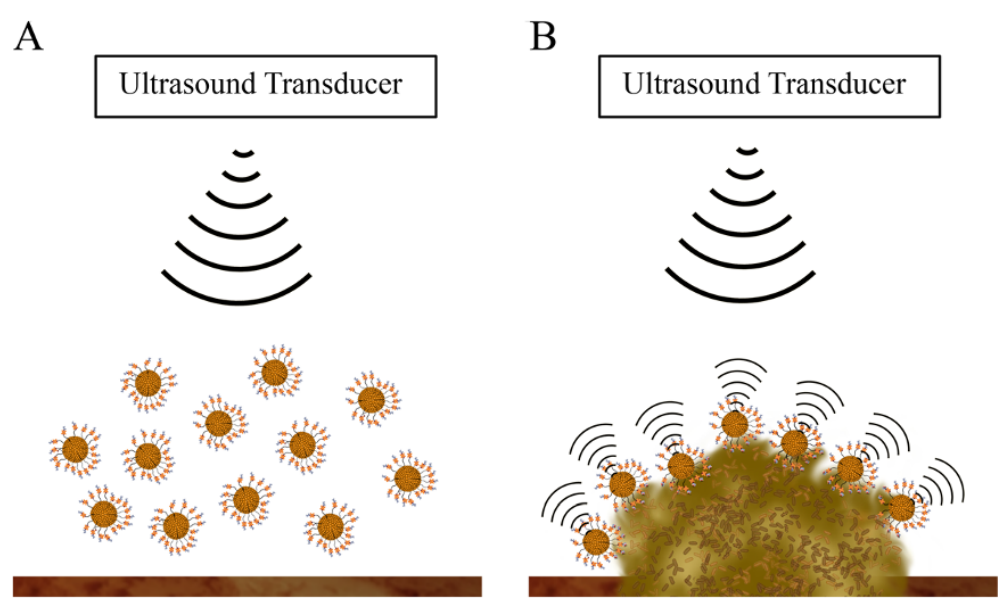

Figure $\mathbf{5}$ Targeted UCAs bind specifically to the biofilm matrix and are detected by ultrasound. (A) Ultrasound insonification of a region where no biofilm matrix is present. The acoustic signature originating from the unbound UCAs will be different than the one from bound UCAs. (B) Biofilm matrix is detected by ultrasound using targeted UCAs that specifically bind to the biofilm matrix.

Biofilms occurring from infections pose a challenge to current medicine because of the difficulty for early detection and diagnosis. Biofilms protect bacteria and promote resistance to antibiotics and chemotherapeutic agents. Moreover, detecting early and late biofilm formation may be problematic due to their dynamic profile. Individual bacterial cells may detach from the biofilm to colonize other niches or an entire biofilm colony may move as a whole across a region [6]. Thus, biofilm-mediated rippling effects that occur during detachment and transmigration pose biomedical challenges. One example is ventilatorassociated pneumonia in immunologically compromised patients that may occur due to biofilm rippling (e.g. cancer patients) [41]. Our data indicates that the binding efficiency of UCAs correlates with matrix biomass. Thus, we propose that the rolling and rippling effects observed during biofilm maturation may reduce biomass and therefore decrease imaging capabilities at the late stages of biofilm matrix formation. It may be that there is a critical timeframe where UCAs bind well and imaging is enhanced and that as the biofilm grows, detaches and ripples, binding is decreased. Developing better detection methodologies and hence diagnostic clinical imaging methods are needed to assess biofilm formation early. By detection of biofilm infections at their earlier stages, this method will potentially offer more treatment options.

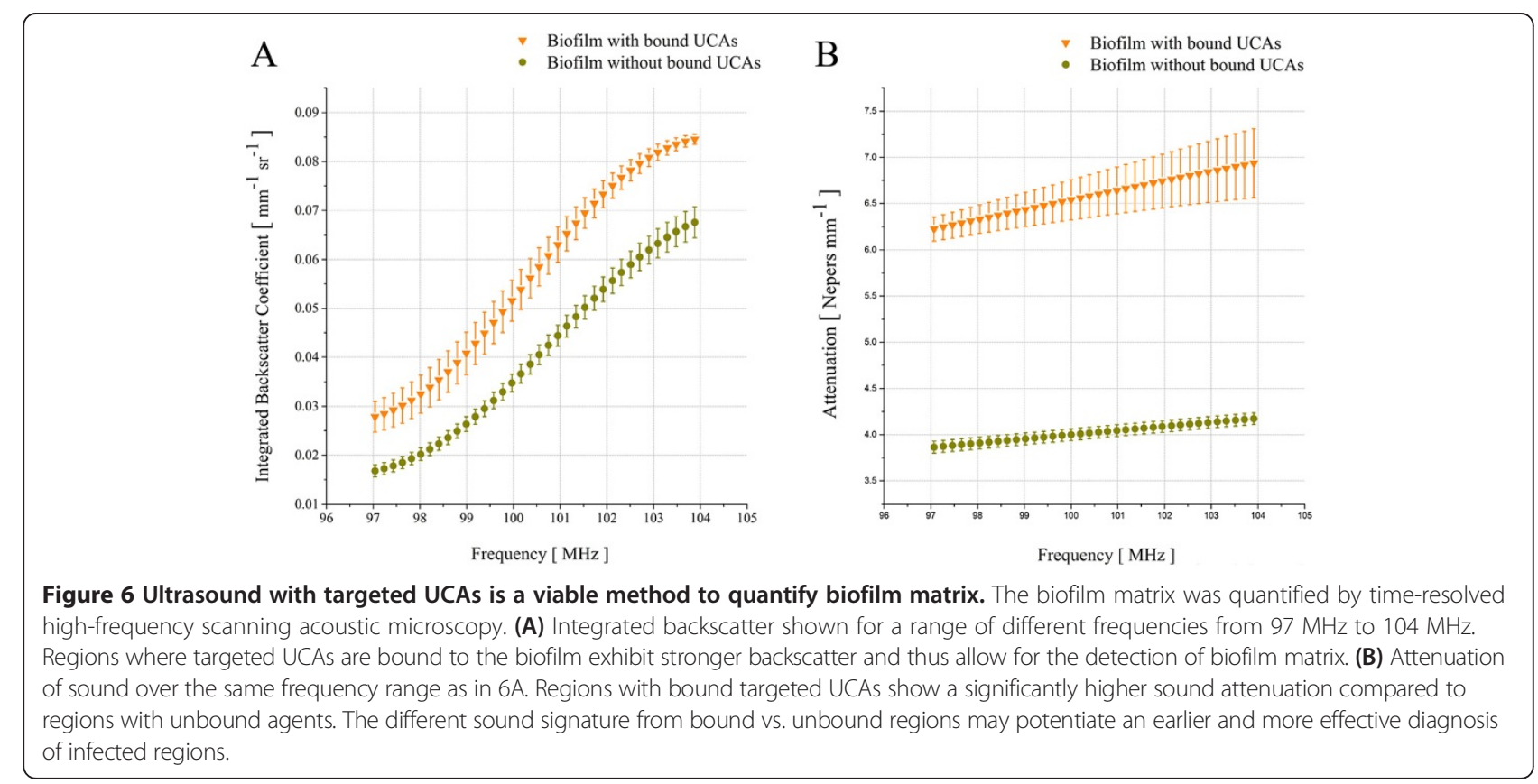


Due to the complex structure of biofilm matrices, we focused on the lectins concanavalin A and WGA $[5,42]$ because biofilms may switch between these two polysaccharides during growth (Table 3). Targeting carbohydrate epitopes that are present in early biofilm matrices may provide novel biofilm markers that will enhance a more optimal molecular imaging, particularly at early stage formation.

Ultrasound imaging devices are readily available in clinical settings and the application of ultrasound techniques for biofilm-relevant infections is familiar to hospital personnel for diseases such as infective endocarditis [12,23,43] and cancer [44]. Targeted UCAs have the potential to recognize and bind to early stage biofilm matrix and thus, facilitate an early diagnosis. Our study demonstrated that while more targeted UCAs bound to larger biofilm matrix mass, a significant number of targeted UCAs also bound to less developed biofilm matrices. Targeted UCAs with ultrasound imaging may provide a means for early detection of biofilm formation within a non-invasive setting. This would include detecting endocarditis and biofilm matrix formation at the site of medical implants such as prosthetic devices, and catheters. Moreover, cancer patients rely on catheterization for chemotherapy treatment and biofilms are prevalent at the catheter interface. In immunologically compromised patients, rippling effects of the late stage biofilms have been reported to promote biofilm transmigration to the lungs causing additional complications in treatment $[45,46]$. Thus, the use of targeted UCAs may provide a rapid method to facilitate early diagnosis in a number of diseases.

High-frequency ultrasound may be used to assess biofilm development in vitro. Currently clinical biomicroscopy uses frequencies in the range of $15-50 \mathrm{MHz}$ including intravascular ultrasound spectroscopy (IVUS), cardiovascular and ocular applications [47-54]. In particular, clinical imaging applications, such as detecting metastases in the eye, are in the range of 15-50 MHz, although research has been performed to measure at the higher frequency of $75 \mathrm{MHz}$ [50]. The use of higher frequencies in the range of $100 \mathrm{MHz}$ has been used for the imaging of choroidal metastasis [55]. Clinical ultrasound applications have focused on higher frequencies in the range of $100-200 \mathrm{MHz}$ [56]. Low resonance frequencies are used clinically for drug delivery applications in conjunction with UCAs and targeted drug delivery as these low frequencies induce microbubble rupture [57]. Thus, in terms of imaging the higher frequencies provide enhanced imaging capabilities whereas lower resonant frequencies allow for more efficient targeted drug delivery. We report for the first time a method to quantify backscatter intensity and mechanoelastic properties of biofilms [58-64]. With regard to the integrated backscatter and the acoustic attenuation, considering differences in the frequency domain, similar values have been previously reported for cancer cells and tissues [58,61,63-66]. A more in-depth understanding of the three-dimensional biofilm matrix structural and mechanoelastic parameters will enhance biofilm imaging and subsequent treatment. Targeted UCAs potentially provide a novel means of imaging for the diagnosis of biofilm infections in vivo.

\section{Materials and methods}

\section{Bacterial strains and cultivation of biofilms}

We used a penicillin-resistant mutant of $S$. aureus. $S$. aureus and coagulase-negative staphylococci account for the majority of device-related infections [67].

S. aureus cultures were stored frozen at $-80^{\circ} \mathrm{C}$ in $10 \%$ glycerol and 90\% tryptic soy broth (TSB, T8907, SigmaAldrich, St. Louis, USA) solution dissolved in sterile ultrapure water (Alfa Aesar, Ward Hill, MA, USA).

A vial of frozen bacterial culture was thawed at room temperature (RT) and added to $250 \mathrm{~mL}$ of TSB. The inoculum was propagated and incubated overnight on an incubator shaker at $37^{\circ} \mathrm{C}$ and 160 rotations per minute (RPMs). The bacterial cultures were harvested after standardization to an optical density at $600 \mathrm{~nm}\left(\mathrm{OD}_{600}\right)$ of 0.05 relative to the TSB culture medium (Beckman Coulter, Inc., Fullerton, CA, USA).

Biofilm assays were conducted by adding three milliliters of the standardized bacterial culture solution to the pretreated $35 \mathrm{~mm}$ glass (World Precision Instruments, Inc., Sarasota, FL, USA) and polystyrene petri dishes (Greiner Bio-One, Monroe, NC, USA). Glass and polystyrene petri dishes were treated in a previous step with Collagen IV (BD Biosciences, San Jose, CA, USA) for twenty minutes and rinsed in three washing steps with sterile distilled water. Prior to the addition of the inoculum, a $22 \times 22 \mathrm{~mm}$ sterile micro cover glass (VWR International, LLC, West Chester, PA, USA) was placed into each of the polystyrene petri dishes. The glass and polystyrene petri dishes were then kept inside an incubator shaker at $37^{\circ} \mathrm{C}$ and 120 RPMs for up to 96 hours without replacement and addition of fresh culture medium in the interim.

\section{Lectins, antibodies and immunofluorescence}

Fluorescently-labeled lectins, concanavalin A (conA; binds to $\alpha$-Man, $\alpha$-Glc) $[5,42]$ and wheat germ agglutinin

Table 3 Carbohydrate-binding specificity of lectins employed for staining of S. aureus biofilms

\begin{tabular}{lccc}
\hline Lectin (source) & Abbreviation & Conjugate & Main specificity \\
\hline Concanavalin A (Canavalia ensiformis) & ConA & FITC, TRITC & a-Man, a-GlC \\
Wheat germ agglutinin (Triticum vulgaris) & WGA & FITC, TRITC & (B-GICNAC) 2, NeuNAC \\
\hline
\end{tabular}


(WGA; binds to ( $\beta$-GlcNAc) ${ }_{2}$ and NeuNAc; SigmaAldrich Corp., St. Louis, MO, USA) [5,42] conjugated with FITC were used for the visualization of carbohydratecontaining extracellular polymeric substances in biofilms of $S$. aureus (Table 3) [5,42]. Stock solution of ConA at a concentration of $1 \mathrm{mg} / \mathrm{mL}$ in $0.1 \mathrm{M}$ sodium bicarbonate $(\mathrm{pH}$ 8.3) and WGA at a concentration of $1 \mathrm{mg} / \mathrm{mL}$ in phosphate buffered saline (PBS; pH 7.4) were prepared, aliquoted and stored at $-20^{\circ} \mathrm{C}$. Prior to use, thawed portions of ConA and WGA aliquots were diluted with $0.1 \mathrm{M}$ sodium bicarbonate $(\mathrm{pH}$ 8.3) and PBS ( $\mathrm{pH} 7.4)$ respectively to a lectin final concentration of $10 \mu \mathrm{g} / \mathrm{mL}$.

The blue-fluorescent nucleic acid stain 4',6-diamidino-2-phenylindole, dihydrochloride (DAPI, SigmaAldrich Corp., St. Louis, MO, USA) was used to visualize bacterial cell distribution in the biofilms. A DAPI stock solution at a concentration of $5 \mathrm{mg} / \mathrm{mL}$ and $14.3 \mathrm{mM}$ in ultrapure water was prepared, aliquoted and stored at $-20^{\circ} \mathrm{C}$. An aliquot was diluted to $300 \mathrm{nM}$ in PBS immediately before use.

The monoclonal immunoglobulin antibody to protein A of $S$. aureus was used as a conjugation agent for the UCA particles. Anti-Protein A (APA) was developed in rabbit using protein A purified from $S$. aureus (SigmaAldrich Corp., St. Louis, MO, USA). Protein A localizes on the surface of staphylococcal bacterial strains and its distribution is inhomogeneous $[68,69]$. The lyophilized content of the vial was reconstituted in $2 \mathrm{~mL}$ PBS $(\mathrm{pH} 7.4)$ yielding a solution with a protein concentration of $23.7 \mathrm{mg} / \mathrm{mL}$. The lectin from P. aeruginosa (PAIL, Sigma-Aldrich Corp., St. Louis, MO, USA) was used, similarly to APA, to conjugate the surface of the UCA particles. The lyophilized content was diluted in $1 \mathrm{~mL}$ PBS ( $\mathrm{pH} 7.4$ ) yielding a protein concentration of $1 \mathrm{mg} / \mathrm{mL}$. Following the reconstitution of APA and PAIL, the proteins were biotinylated and conjugated onto the surface of the UCAs according to the method that will be described in more detail.

Sulfo-NHS-LC-Biotin (Thermo Scientific, Rockford, IL, USA) was applied to label APA and PA-IL with biotin. The vial of Sulfo-NHS-LC-Biotin was stored at $-20^{\circ} \mathrm{C}$ and equilibrated to $\mathrm{RT}$ before opening to avoid condensation. For the biotin labeling reaction $2.2 \mathrm{mg}$ of Sulfo-NHS-LC-Biotin were dissolved in $400 \mu \mathrm{L}$ ultrapure water immediately before use yielding a $10 \mathrm{mM}$ solution. A 20-fold molar excess of biotin reagent to label APA and PA-IL, resulting in 4-6 biotin groups per antibody molecule, was found to be suitable. For APA with a concentration of $23.7 \mathrm{mg} / \mathrm{mL}$, a volume of $320 \mu \mathrm{L}$ Sulfo-NHS-LC-Biotin was used for the biotinylation reaction while $13.5 \mu \mathrm{L}$ Sulfo-NHS-LC-Biotin were used to label PA-IL with a concentration of $1 \mathrm{mg} /$ $\mathrm{mL}$. Following the incubation on ice for two hours at RT a Zeba ${ }^{\oplus}$ desalt spin column (Thermo Scientific,
Rockford, IL, USA) was applied to remove the excess non-reacted and hydrolyzed Sulfo-NHS-LC-Biotin reagent from the APA and PA-IL solutions. The column was placed into a sterile $15 \mathrm{~mL}$ falcon tube and centrifuged at $1000 \times \mathrm{G}$ for two minutes. After centrifugation the storage buffer collected at the bottom of the falcon tube was discarded, the column placed back into the same falcon tube and equilibrated by adding $2.5 \mathrm{~mL}$ of PBS (Thermo Scientific, Rockford, IL, USA) to the top of the resin bed and centrifuging at $1000 \times \mathrm{G}$ for two minutes. Next, the flow-through was discarded and the same step was repeated for a total of three times. Subsequently the column was placed into a new sterile $15 \mathrm{~mL}$ falcon tube and the antibody solution was applied onto the center of the resin bed. Finally, the column was centrifuged at $1000 \times \mathrm{G}$ for two minutes. The collected purified flow-through antibody solutions were aliquoted and stored appropriately.

\section{Targeted ultrasound contrast agents}

Biotin-conjugated lipid-encapsulated perfluorocarbon UCAs with a mean diameter of $3.02 \mu \mathrm{m} \pm 0.05 \mu \mathrm{m}$ (Targeson, San Diego, CA, USA) were removed from a sealed vial using a four-way stopcock-syringe combination with a $22 \mathrm{G}$ needle while simultaneously venting the vial with an additional needle. Using a $22 \mathrm{G}$ needle, targestar conjugation buffer (TCB, Targeson, San Diego, CA, USA) was withdrawn into the syringe containing the UCA particles to a total volume of $3.5 \mathrm{~mL}$ and centrifuged at $400 \times \mathrm{G}$ for three minutes to remove excess free unincorporated lipids from the UCA particle solution. After centrifugation the infranatant was drained drop-wise and the UCAs were re-suspended in $1.0 \mathrm{~mL}$ TCB. Afterward, UCAs were incubated with $150 \mu \mathrm{L}$ FITC-streptavidin (Invitrogen, Carlsbad, CA, USA) at a concentration of $1 \mathrm{mg} / \mathrm{mL}$ for twenty minutes at RT with occasional gentle shaking of the vial. The unreacted FITC-streptavidin was removed in a centrifugal washing at $400 \times \mathrm{G}$ for three minutes similarly to the previous step and re-suspended in $1.0 \mathrm{~mL}$ TCB. Finally, the UCA particles were incubated on ice with either APA or PA-IL for 30 minutes while the unconjugated targeting antibody and ligand molecules were removed by a final centrifugal washing as was described in the previous steps.

\section{Lectin-staining for biofilms}

For the $S$. aureus biofilm matrix, a double staining approach with ConA and WGA was chosen [5,42]. After incubation for twenty minutes in the dark at RT, excess staining solution was removed by rinsing three times with sterile distilled water. 


\section{Epifluorescence microscopy}

Epifluorescence microscopy was carried out with a Zeiss Axioskop 2 microscope equipped with an AxioCam MRc Rev 3. Negative and positive controls were conducted using epifluorescence microscopy. For each case four biofilm samples were used to image five random positions on every sample. Each position was imaged by applying the respective filter for FITC, TRITC and DAPI. The negative control did not use any dyes to control for any possible autofluorescence effects.

\section{Ultrasonic investigation of biofilms}

Ultrasonic imaging and RF data acquisition was performed with a high-frequency scanning acoustic microscope (Fraunhofer IBMT, St. Ingbert, Germany). A detailed description of the acoustic lens used is shown in Table 2.

The recorded RF data were stored for further processing. The post-processing was conducted using customwritten scripts in MATLAB (The Math-Works, Natick, MA, USA). The scripts were applied for the visual reconstruction in $2 \mathrm{D}$ and $3 \mathrm{D}$ of the raw $\mathrm{RF}$ data for the selected ROIs. RF raw signals were gated by applying a rectangular window function. The window length at $100 \mathrm{MHz}$ excitation center frequency was set such as it corresponded to 10 wavelengths. The wavelength estimation is based on the center frequency of the lens $(100 \mathrm{MHz})$.

The gating of RF raw time-signals allows estimations of scattering properties to be related to distinct ROIs in the volume under interrogation [70]. However, the gating process also allows unwanted frequency content to be added into the backscattered power spectrum which subsequently, leads to inaccurate estimates of scatterer properties. In order to minimize such unwanted effects, a Hamming window was applied [70]. The tapered windows reduced the high-frequency content added into the gated RF time-signals by smoothing the edges.

When the acoustic lens is moved over a ROI where the substrate is covered by an EPS layer under investigation, two echoes are received. One echo originates from the top surface of the layer, $S_{0}(t)$, and the second, $S(t)$, from the interface between the layer and the substrate. These signals can be written as follows [71-74]:

$$
\begin{gathered}
S_{0}(t)=A_{0} s\left(t-t_{0}\right) \otimes g\left(t, z_{0}\right) \\
S(t)=A_{1} s\left(t-t_{1}\right) \otimes g\left(t, z_{1}\right)+A_{2} s\left(t-t_{2}\right) \otimes g\left(t, z_{2}\right)
\end{gathered}
$$

$\mathrm{S}(\mathrm{t})$ is the reflected signal from the top and the sample-substrate interface of the EPS matrix. Provided that the defocus is positive meaning that the acoustic lens is elevated above the maximum focus position, it is adequate to constrain the function $g$ to be independent of $\mathrm{t}$ and to be a real function of $\mathrm{z}$ only. The optimum value of $\mathrm{z}$ was found experimentally, by scanning along the $\mathrm{z}$ axis and finding the minimum positive value at which the shape of the waveform remained approximately constant as a function of $\mathrm{z}$. The $\mathrm{z}$ value was experimentally determined to be $900 \mu \mathrm{m}$. Within the approximation of the independence of the waveform shape on $z$, the signals can be written respectively as [74]:

$$
\begin{gathered}
S_{0}(t)=A_{0} s\left(t-t_{0}\right) \times g\left(z_{0}\right) \\
S(t)=A_{1} s\left(t-t_{1}\right) \times g\left(t, z_{1}\right)+A_{2} s\left(t-t_{2}\right) \times g\left(z_{2}\right)
\end{gathered}
$$

From the height in amplitude and position on the time axis of each maximum, the following parameters were measured:

$$
\begin{aligned}
& \Delta \mathrm{T}_{0 / 1}=t_{0}-t_{1} \\
& \Delta \mathrm{T}_{0 / 1}=t_{0}-t_{1}
\end{aligned}
$$

where $t_{1}$ and $t_{2}$ are the arrival times of the sample and the interface echo respectively (Figure $3 \mathrm{~A}$ ) and $t_{0}$ is the time arrival of the reference signal (not shown) when no sample is placed in between the acoustic lens and the substrate. The velocity of the coupling medium, which in this case was degassed biofilm medium at $25^{\circ} \mathrm{C}$, was approximated to the velocity of distilled water and set to be $1497 \mathrm{~m} / \mathrm{s}$ [75,76] while the attenuation of the same medium was set equal to the attenuation of distilled water at $25^{\circ} \mathrm{C}, 2 \mathrm{~dB} / \mathrm{mm}$ [77]. The density of the medium was calculated with a microbalance and a micropipette at $25^{\circ} \mathrm{C}$. From the density of the coupling medium, denoted as $\rho_{c m}$, and the respective ultrasonic velocity, denoted as $v_{c m}$, the acoustic impedance, $Z_{c m}$, of the coupling medium was deduced:

$$
Z_{c m}=\rho_{c m} \times \mathrm{V}_{\mathrm{cm}}
$$

From the difference in time between the reference signal, $t_{0}$, and the reflection from the sample surface, $t_{1}$, and by applying the velocity, $\mathrm{v}_{0}$, of the coupling medium, the thickness of the layer is:

$$
\mathrm{d}=\frac{1}{2}\left(\mathrm{t}_{0}-\mathrm{t}_{1}\right) \mathrm{V}_{0}
$$

From the ratio of magnitude of the reflection $A_{1}$ from the surface of the layer to the magnitude of the reference signal $A_{0}$, and by applying the impedance $Z_{0}$ of the coupling medium as has been calculated in and the acoustic impedance of the substrate $Z_{s}$, the acoustic impedance of the biofilm sample is:

$$
\mathrm{Z}_{\mathrm{bf}}=\mathrm{Z}_{0} \frac{\mathrm{A}_{0}+\mathrm{A}_{1}}{\mathrm{~A}_{0}-\mathrm{A}_{1}}
$$

Finally, from the amplitude $A_{2}$ of the echo from the interface between the layer and the substrate, the 
amplitude of the substrate echo $\mathrm{A}_{0}$, the attenuation in the cell, in units of Nepers per unit length, can be calculated as follows:

$$
\alpha=\alpha_{0}+\frac{1}{2 \mathrm{~d}} \log _{\mathrm{e}}\left(\frac{\mathrm{A}_{0}}{\mathrm{~A}_{2}} \frac{\mathrm{Z}_{\mathrm{s}}-\mathrm{Z}_{\mathrm{bf}}}{\mathrm{Z}_{\mathrm{s}}+\mathrm{Z}_{\mathrm{bf}}} \frac{4 \mathrm{Z}_{\mathrm{c}} \times \mathrm{Z}_{0}}{\left(\mathrm{Z}_{\mathrm{c}}+\mathrm{Z}_{0}\right)^{2}} \frac{\mathrm{Z}_{\mathrm{s}}+\mathrm{Z}_{0}}{\mathrm{Z}_{\mathrm{s}}-\mathrm{Z}_{0}}\right)
$$

\section{Abbreviations}

APA: Anti-Protein A; DAPI: 4',6-diamidino-2-phenylindole, dihydrochloride; IBSC: Integrated backscatter coefficient; FITC: Fluorescein isothiocyanate; OD: Optical density; PBS: Phosphate buffered saline; RF: Radio-frequency; ROI: Region of interest; RPM: Revolutions per minute; RT: Room temperature; StDev: Standard deviation; TCB: Targeson conjugation buffer;

TRITC: Tetramethylrhodamine isothiocyanate; TSB: Tryptic soy broth.

\section{Competing interests}

The authors declare that they have no competing interests.

\section{Authors' contributions}

PA, KDAM, JSA, MLM: Designed the study. PA, KDAM: Conducted the experiments, performed data analysis, performed statistical analysis. PA MLM: Prepared and edited the manuscript. JSA, KDAM: Edited the manuscript. All authors added intellectual content, read and approved the final version.

\section{Acknowledgements}

We thank Dr. Tung T. Hoang (Microbiology Dept., University of Hawaii at Manoa) for providing us with the bacterial strain. We would like to thank Dr. Terry Matsunaga (Dept. of Radiology, University of Arizona, Tucson, AZ, USA), Dr. Joshua Rychak (Targeson, San Diego, CA, USA) and Dr. Alexander L. Klibanov (Dept. of Biomedical Engineering, University of Virginia,

Charlottesville, VA, USA) for sharing their technical expertise with us and for valuable discussions. This work was supported by: National Institutes of Health (NCRR P20-RR016453 and RO1GM104984 to M.L.M).

\section{Author details}

${ }^{1}$ University of Hawaii Cancer Center, Honolulu, HI 96813, USA. ${ }^{2}$ Molecular Biosciences and Bioengineering, University of Hawaii at Manoa, Honolulu, $\mathrm{HI}$ 96822, USA. ${ }^{3}$ Mechanical Engineering, University of Hawaii at Manoa, Honolulu, HI 96822, USA. "Department of Oceanography, School of Ocean and Earth Sciences and Technology, University of Hawaii at Manoa, Honolulu, HI, USA. ${ }^{5}$ Current address: Department of Biological Oceanography, Royal Netherlands Institute for Sea Research (NIOZ), P.O. Box 59, 1790 AB Den Burg, Texel, The Netherlands.

Received: 6 March 2014 Accepted: 24 June 2014 Published: 6 July 2014

\section{References}

1. Liu D, Lau YL, Chau YK, Pacepavicius G: Simple technique for estimation of biofilm accumulation. Bull Environ Contam Toxicol 1994, 53(6):913-918.

2. Allison DG, Ruiz B, SanJose C, Jaspe A, Gilbert P: Extracellular products as mediators of the formation and detachment of Pseudomonas fluorescens biofilms. Fems Microbiol Letters 1998, 167(2):179-184.

3. Costerton JW, Stewart PS, Greenberg EP: Bacterial biofilms: a common cause of persistent infections. Science 1999, 284(5418):1318-1322.

4. Wingender J, Neu TR, Flemming HC: What are Bacterial Extracellular Substances? In Microbial Extracellular Polymeric Substances: Characterization, Structure and Function. 1st edition. Edited by Wingender J, Neu TR, Flemming HC. Berlin: Springer; 1999:1-19.

5. Strathmann M, Wingender J, Flemming HC: Application of fluorescently labelled lectins for the visualization and biochemical characterization of polysaccharides in biofilms of pseudomonas aeruginosa. J Microbiol Methods 2002, 50(3):237-248.

6. Hall-Stoodley L, Costerton JW, Stoodley P: Bacterial biofilms: from the natural environment to infectious diseases. Nat Rev Microbiol 2004 2(2):95-108.

7. Davey ME, GA O'T: Microbial biofilms: from ecology to molecular genetics. Microbiol Mol Biol Rev 2000, 64(4):847-867.
8. Flemming HC, Wingender J: The biofilm matrix. Nat Rev Microbio/ 2010, 8(9):623-633.

9. Lambe DW, Ferguson KP, Mayberry-Carson KJ, Tober-Meyer B, Costerton JW: Foreign-body-associated experimental osteomyelitis induced with bacteroides fragilis and staphylococcus epidermidis in rabbits. Clin Orthop Relat Res 1991, 266:285-294.

10. Harris LG, Richards RG: Staphylococci and implant surfaces: a review. Injury-Int J Care Injured 2006, 37:3-14.

11. Baldassarri L, Montanaro L, Creti R, Arciola CR: Underestimated collateral effects of antibiotic therapy in prosthesis-associated bacterial infections. Int J Artif Organs 2007, 30:786-791.

12. Kaplan JB: Methods for the treatment and opinion prevention of bacterial biofilms. Expert Opinion Therap Patents 2005, 15(8):955-965.

13. Sullam PM, Drake TA, Sande MA: Pathogenesis of endocarditis. Am J Med 1985, 78(6B):110-115.

14. Caldwell DA, Lovasik D: Endocarditis in the immunocompromised. Am J Nurs 2002, (Suppl):32-36.

15. Donlan RM, Costerton JW: Biofilms: survival mechanisms of clinically relevant microorganisms. Clin Microbiol Rev 2002, 15(2):167-193.

16. Petti CA, Fowler VG Jr: Staphylococcus aureus bacteremia and endocarditis. Cardiol Clin 2003, 21(2):219-233. vii.

17. Nickel JC, Wright JB, Ruseska I, Marrie TJ, Whitfield C, Costerton JW: Antibiotic-resistance of pseudomonas-aeruginosa colonizing a urinary catheter invitro. Eur J Clin Microbiol Infect Dis 1985, 4(2):213-218.

18. Allison DG, Gilbert P: Modification by surface association of antimicrobial susceptibility of bacterial-populations. J Ind Microbiol 1995, 15(4):311-317.

19. Stewart PS, Costerton JW: Antibiotic resistance of bacteria in biofilms. Lancet 2001, 358(9276):135-138.

20. Parsek MR, Singh PK: Bacterial biofilms: an emerging link to disease pathogenesis. Annu Rev Microbiol 2003, 57:677-701.

21. Furuya EY, Lowy FD: Antimicrobial strategies for the prevention and treatment of cardiovascular infections. Curr Opin Pharmacol 2003, 3(5):464-469.

22. Durack DT, Lukes AS, Bright DK, Alberts MJ, Bashore TM, Corey GR, Douglas JM, Gray L, Harrell FE, Harrison JK, Heinle SA, Morris A, Kisslo JA, Nicely LM, Oldham N, Penning LM, Sexton DJ, Towns M, Waugh RA: New criteria for diagnosis of infective endocarditis - utilization of specific echocardiographic findings. Am J Med 1994, 96(3):200-209.

23. Baddour LM, Bettmann MA, Bolger AF, Epstein AE, Ferrieri $P$, Gerber $M A$, Gewitz MH, Jacobs AK, Levison ME, Newburger JW, Pallasch TJ, Wilson WR, Baltimore RS, Falace DA, Shulman ST, Tani LY, Taubert KA: Infective endocarditis: diagnosis, antimicrobial therapy, and management of complications. Circulation 2005, 112(15):2373.

24. Shemesh H, Goertz DE, van der Sluis LW, de Jong N, Wu MK, Wesselink PR High frequency ultrasound imaging of a single-species biofilm. J Dent 2007, 35(8):673-678.

25. Vaidya K, Osgood R, Ren D, Pichichero ME, Helguera M: Ultrasound imaging and characterization of biofilms based on wavelet de-noised radiofrequency data. Ultrasound Med Biol 2014, 40(3):583-595.

26. Good MS, Wend CF, Bond U, McLean JS, Panetta PD, Ahmed S, Crawford SL, Daly DS: An estimate of biofilm properties using an acoustic microscope. IEEE Trans Ultrason Ferroelectr Frea Control 2006, 53(9):1637-1646.

27. Holmes AK, Laybourn-Parry J, Parry JD, Unwin ME, Challis RE: Ultrasonic imaging of biofilms utilizing echoes from the biofilm/air interface. IEEE Trans Ultrason Ferroelectr Freq Control 2006, 53(1):185-192.

28. Kujundzic E, Fonseca AC, Evans EA, Peterson M, Greenberg AR, Hernandez M: Ultrasonic monitoring of early-stage biofilm growth on polymeric surfaces. J Microbiol Methods 2007, 68(3):458-467.

29. Sbeity F, Menigot S, Charara J, Girault JM: Contrast improvement in sub- and ultraharmonic ultrasound contrast imaging by combining several hammerstein models. Int J Biomed Imag 2013, 2013:270523.

30. Anderson CR, Hu X, Zhang H, Tlaxca J, Decleves A-E, Houghtaling R, Sharma K, Lawrence M, Ferrara KW, Rychak JJ: Ultrasound molecular imaging of tumor angiogenesis with an integrin targeted microbubble contrast agent. Investig Radiol 2011, 46(4):215-224.

31. Klibanov AL: Microbubble contrast agents - targeted ultrasound imaging and ultrasound-assisted drug-delivery applications. Investig Radio/ 2006, 41(3):354-362

32. Klibanov AL: Ultrasound molecular imaging with targeted microbubble contrast agents. J Nucl Cardiol 2007, 14(6):876-884. 
33. Klibanov AL: Preparation of targeted microbubbles: ultrasound contrast agents for molecular imaging. Med Biol Eng Comput 2009, 47(8):875-882.

34. Klibanov AL, Hughes MS, Villanueva FS, Jankowski RJ, Wagner WR, Wojdyla JK, Wible $\mathrm{JH}$, Brandenburger $\mathrm{GH}$ : Targeting and ultrasound imaging of microbubble-based contrast agents. Magnet Reson Mat Biol Physics Med 1999, 8(3):177-184.

35. Unnikrishnan S, Klibanov AL: Microbubbles as ultrasound contrast agents for molecular imaging: preparation and application. Am J Roentgenol 2012, 199(2):292-299.

36. Weiss EC, Anastasiadis P, Pilarczyk G, Lernor RM, Zinin PV: Mechanical properties of single cells by high-frequency time-resolved acoustic microscopy. Ultrason Ferroelectr Frequen Contr IEEE Trans 2007, 54(11):2257-2271.

37. Weiss EC, Lemor RM, Pilarczyk G, Anastasiadis P, Zinin PV: Imaging of focal contacts of chicken heart muscle cells by high-frequency acoustic microscopy. Ultrasound Med Biol 2007, 33(8):1320-1326.

38. Zinin PV, Allen JS III: Deformation of biological cells in the acoustic field of an oscillating bubble. Phys Rev E 2009, 79(2 Pt 1):021910.

39. Zinin PV, Allen JS 3rd, Levin VM: Mechanical resonances of bacteria cells. Phys Rev E Stat Nonlin Soft Matter Phys 2005, 72(6 Pt 1):061907.

40. Moran CM, Watson RJ, Fox KAA, McDicken WN: In vitro acoustic characterisation of four intravenous ultrasonic contrast agents at $30 \mathrm{MHz}$. Ultrasound Med Biol 2002, 28(6):785-791.

41. Inglis TJ: Evidence for dynamic phenomena in residual tracheal tube biofilm. Br J Anaesth 1993, 70(1):22-24.

42. Goldstein IJ, Hayes CE: The lectins: carbohydrate-binding proteins of plants and animals. Adv Carbohydr Chem Biochem 1978, 35:127-340.

43. Archibald LK, Gaynes RP: Hospital-acquired infections in the United States. The importance of interhospital comparisons. Infect Dis Clin North Am 1997, 11(2):245-255.

44. Azzopardi EA, Ferguson EL, Thomas DW: The enhanced permeability retention effect: a new paradigm for drug targeting in infection. J Antimicrob Chemother 2013, 68(2):257-274.

45. Inglis TJJ, Titmeng L, Mahlee N, Eekoon T, Kokpheng $\mathrm{H}$ : Structural features of tracheal tube biofilm formed during prolonged mechanical ventilation. Chest 1995, 108(4):1049-1052.

46. Al Akhrass F, Al Wohoush I, Chaftari AM, Reitzel R, Jiang Y, Ghannoum M, Tarrand J, Hachem R, Raad I: Rhodococcus bacteremia in cancer patients is mostly catheter related and associated with biofilm formation. Plos ONE 2012, 7(3):e32945.

47. Hejsek L, Pasta J: Ultrasonographic biomicroscopy of the eye. Ultrazvukova? Biomikroskopie oka 2005, 61(4):273-280.

48. Liang HD, Blomley MJK: The role of ultrasound in molecular imaging Br J Radiol 2003, 76(suppl_2):S140-S150.

49. Silverman $\mathrm{RH}$ : High-resolution ultrasound imaging of the eye - a review. Clinical Exper Ophthalmol 2009, 37(1):54-67.

50. Silverman RH, Cannata J, Shung KK, Gal O, Patel M, Lloyd HO, Feleppa EJ, Coleman DJ: $75 \mathrm{MHz}$ ultrasound biomicroscopy of anterior segment of eye. Ultrason Imaging 2006, 28(3):179-188

51. Snetkova $Y$, Levin VM, Petriniuk YS, Denisov AF, Bogachenkov AN, Denisova LA, Khramtzova YA: Application of the method of acoustic microscopy for the study of eye tissues. Morfologiya 2005, 127(2):72-75.

52. Goertz DE, Frijlink ME, De Jong N, Steen A: Nonlinear intravascular ultrasound contrast imaging. Ultrasound Med Biol 2006, 32(4):491-502.

53. Goertz DE, Frijlink ME, Tempel D, Bhagwandas V, Gisolf A, Krams R, de Jong N, van der Steen AFW: Subharmonic contrast intravascular ultrasound for vasa vasorum imaging. Ultrasound Med Biol 2007, 33(12):1859-1872.

54. Phillips LC, Klibanov AL, Wamhoff BR, Hossack JA: Intravascular ultrasound detection and delivery of molecularly targeted microbubbles for gene delivery. IEEE Trans UItrason Ferroelectr Frea Control 2012, 59(7):1596-1601.

55. Witkin AJ, Fischer DH, Shields CL, Reichstein D, Shields JA: Enhanced depth imaging spectral-domain optical coherence tomography of a subtle choroidal metastasis. Eye 2012, 26(12):1598-1599.

56. Knspik DA, Starkoski B, Pavlin CJ, Foster FS: A 100-200 MHz ultrasound biomicroscope. IEEE Trans UItrason Ferroelectr Freq Control 2000, 47(6):1540-1549.

57. Liu Y, Miyoshi H, Nakamura M: Encapsulated ultrasound microbubbles: therapeutic application in drug/gene delivery. J Control Release 2006, 114(1):89-99.

58. Saijo Y, Sasaki H, Okawai H, Nitta S-I, Tanaka M: Acoustic properties of atherosclerosis of human aorta obtained with high-frequency ultrasound. Ultrasound Med Biol 1998, 24(7):1061-1064.
59. Saijo $Y$, Jorgensen CS, Falk E: Ultrasonic tissue characterization of collagen in lipid-rich plaques in apoE-deficient mice. Atherosclerosis 2001, 158(2):289-295.

60. Saijo Y, Santos E, Sasaki H, Yambe T, Tanaka M, Hozumi N, Kobayashi K, Okada N: Ultrasonic tissue characterization of atherosclerosis by a speed-of-sound microscanning system. IEEE Trans Ultrason Ferroelectr Frequen Contr 2007, 54(8):1571-1577.

61. Saijo Y, Miyakawa T, Sasaki H, Tanaka M, Nitta Sl: Acoustic properties of aortic aneurysm obtained with scanning acoustic microscopy. Ultrasonics 2004, 42(1-9):695-698.

62. Brand S, Solanki B, Foster DB, Czarnota GJ, Kolios MC: Monitoring of cell death in epithelial cells using high frequency ultrasound spectroscopy. Ultrasound Med Biol 2009, 35(3):482-493.

63. Brand S, Weiss EC, Lemor RM, Kolios MC: High frequency ultrasound tissue characterization and acoustic microscopy of intracellular changes. Ultrasound Med Biol 2008, 34(9):1396-1407.

64. Strohm EM, Czarnota GJ, Kolios MC: Quantitative measurements of apoptotic cell properties using acoustic microscopy. IEEE Transactions Ultrason Ferroelectr Frequen Contr 2010, 57(10):2293-2304.

65. Baddour RE, Sherar MD, Hunt JW, Czarnota GJ, Kolios MC: High-frequency ultrasound scattering from microspheres and single cells. J Acoust Soc Am 2005, 117(2):934-943.

66. Saijo Y, Tanaka M, Okawai H, Sasaki H, Nitta SI, Dunn F: Ultrasonic tissue characterization of infarcted myocardium by scanning acoustic microscopy. Ultrasound Med Biol 1997, 23(1):77-85.

67. Baddour LM, Bettmann MA, Bolger AF, Epstein AE, Ferrieri P, Gerber MA, Gewitz MH, Jacobs AK, Levison ME, Newburger JW, Pallasch TJ, Wilson WR, Baltimore RS, Falace DA, Shulman ST, Tani LY, Taubert KA: Nonvalvular cardiovascular device-related infections. Circulation 2003, 108(16):2015-2031.

68. DeDent AC, McAdow M, Schneewind O: Distribution of protein A on the surface of staphylococcus aureus. J Bacterio/ 2007, 189(12):4473-4484.

69. Schneewind O, Fowler A, Faull KF: Structure of the cell wall anchor of surface proteins in staphylococcus aureus. Science 1995, 268(5207):103-106.

70. Oelze ML, O'Brien WD: Improved scatterer property estimates from ultrasound backscatter for small gate lengths using a gate-edge correction factor. J Acoust Soc Am 2004, 116(5):3212-3223.

71. Briggs A: Advances in Acoustic Microscopy. New York: Plenum Press; 1995

72. Briggs A, Kolosov O: Acoustic Microscopy. New York: Clarendon; 2010.

73. Briggs GAD, Rowe JM, Sinton AM, Spencer DS: Quantitative Methods in Acoustic Microscopy. In Ultrasonics Symposium Proceedings: 1988. Chicago, IL, USA: Publ by IEEE; 1988:743-749.

74. Briggs GAD, Wang J, Gundle R: Quantitative acoustic microscopy of individual living human cells. J Microsc 1993, 172(Pt1):3-12.

75. Martin G, Carroll ET: Tables of the speed of sound in water. J Acoustic SoC Am 1959, 31(1):75-76.

76. Greenspan M, Tschiegg CE: Tables of the speed of sound in water. J Acoustic Soc Am 1959, 31(1):75-76.

77. Akashi N, Kushibiki J, Dunn F: Acoustic properties of egg yolk and albumen range 20-400 MHz. J Acoust Soc Am 1997, 102(6):3774-3778.

\section{doi:10.1186/1477-3155-12-24}

Cite this article as: Anastasiadis et al:: Detection and quantification of bacterial biofilms combining high-frequency acoustic microscopy and targeted lipid microparticles. Journal of Nanobiotechnology 2014 12:24.

\section{Submit your next manuscript to BioMed Central and take full advantage of:}

- Convenient online submission

- Thorough peer review

- No space constraints or color figure charges

- Immediate publication on acceptance

- Inclusion in PubMed, CAS, Scopus and Google Scholar

- Research which is freely available for redistribution 